\title{
Stability Analysis for Stochastic Neutral-Type Memristive Neural Networks with Time-Varying Delay and S-Type Distributed Delays
}

\author{
Changjian Wang, Zuoliang Xiong, Min Liang, and Hongwei Yin \\ Department of Math, Nanchang University, Nanchang, Jiangxi Province 330031, China \\ Correspondence should be addressed to Zuoliang Xiong; xiong1601@163.com
}

Received 5 August 2016; Accepted 9 November 2016; Published 30 January 2017

Academic Editor: Qingling Zhang

Copyright ( $(2017$ Changjian Wang et al. This is an open access article distributed under the Creative Commons Attribution License, which permits unrestricted use, distribution, and reproduction in any medium, provided the original work is properly cited.

In this paper, we consider the input-to-stability for a class of stochastic neutral-type memristive neural networks. Neutral terms and S-type distributed delays are taken into account in our system. Using the stochastic analysis theory and Itô formula, we obtain the conditions of mean-square exponential input-to-stability for system. A numerical example is given to illustrate the correctness of our conclusions.

\section{Introduction}

The complex network is considered to be one of the leading research subjects of science and technology in twenty-first Century, which include neural networks, communication networks, power networks, and social networks. In particular, the research on the neural network is very widely including the control theory, stability theory, and bifurcation theory (see [1-4]). As a special case of complex network, memristive neural networks can better simulate the human brain, so it has also become a focus for the majority of scholars. The memristor is a kind of nonlinear resistor which has memory function to simulate the mechanism of human neuron and synapse. Recently, memristive neural networks systems have been successfully applied in associative memory, chaos synchronization, image processing, and so on. Although a lot of achievements have been made in the field of application, most of the current research efforts on memristive neural networks are mainly focused on deterministic models (see [5-7]). However, in reality, noise disturbance always exists, which may cause instability and other poor performances. So it is necessary to study the memristive neural networks with random disturbance due to its theoretical and practical significance.
In addition, in order to deal with the dynamic image, we need to introduce delays between the signal transmissions of neurons, which have formed the memristive neural networks with delays. In practical application, it is more common for a dynamic system with time-varying delay, because the constant delay is only an ideal approximation of the timevarying delay. Many scholars have made great achievements in this respect (see [8-11]). Here, we have to point out that neural networks are composed of a large number of neurons, many of which are clustered into spherical or layered structures and interact with each other and are connected to a variety of complex neural pathways through the axon. Thus, there exists distributed delay in the transmission of signals. Usually, the discrete delays and distributed delays cannot contain each other in the same system; however, in [12] we can see that discrete delays and distributed delays can be written in a unified form under Stieltjes-Lebesgue integral, that is, S-type distributed delays (see $[13,14]$ ). In fact, the differential expression of the systems not only is related to the derivative of the current state but also has a great relationship with the derivative of the past state. It is called neutral delay neural network. Therefore, it is very significant to study the stochastic neutral-type memristive neural network with time-varying delays and S-type distributed delays. 
The control input has a great influence on the dynamic behavior of the neural network. The input-to-state stability (ISS) was first proposed by Sontag to check robust stability, which is more general than the traditional exponential stability. The traditional exponential stability includes asymptotical stability, exponential stability, and almost sure stability. In $[15,16]$, global asymptotical stability analysis for a kind of discrete-time recurrent neural network has been studied. In [17-22], exponential stability and almost sure stability of the neural network have been investigated. As far as we know, the traditional stability is that the state of the neural network is close to the equilibrium point when the time approaches infinity. But this does not always happen in our reality. ISS control analysis opens up a new dynamic neural network application in nonlinear system. In [23], input-tostate stability for a class of stochastic memristive neural networks with time-varying delay has been studied.

Motivated by the above discussion, even though the stability problem of stochastic neural networks has been studied, there are few studies on the stability of stochastic neutraltype memristive neural network. In this paper, we consider the stochastic neutral-type memristive neural networks to end the gap. Using the stochastic analysis theory and Itô formula, we obtain the sufficient conditions of mean-square exponential input-to-stability and some corollaries for system (1).

The rest of the paper is organized as follows. In Section 2, we present a model and give some hypotheses. The main conclusions are proved in Section 3. In Section 4, a numerical example is given to illustrate the correctness of our conclusions. Finally, the further discussion is drawn in Section 5.

Throughout this paper, solutions of all the systems considered are intended in the Filippovs sense. Let $R^{n}$ represent $n$-dimensional Euclidean space. The superscript " $T$ " denotes the transpose of a matrix or vector. $l_{\infty}$ denotes the class of essentially bounded functions $u$ from $[0,+\infty]$ to $R^{n}$ with $\|u\|_{\infty}=\operatorname{esssup}_{t \geq 0}|u|<\infty$. Let $\tau>0$ and $C\left([-\tau, 0], R^{n}\right)$ denote the family of continuous functions $\varphi$ from $[-\tau, 0]$ to $R^{n}$ with the norm $\|\varphi\|=\sup _{-\tau \leq s \leq 0}|\varphi(s)|$, where $|\cdot|$ is the Euclidean norm in $R^{n}$. Let $L_{F_{0}}^{2}\left([-\tau, 0] ; R^{n}\right)$ denote the family of all $F_{0}$ measurable, $\ell\left([-\tau, 0] ; R^{n}\right)$-valued stochastic variables $\psi=\{\psi(s):-\tau \leq s \leq 0\}$ such that $\int_{-\tau}^{0} E\left[|\psi(s)|^{2}\right] d s<\infty$, where $E[\cdot]$ stands for the correspondent expectation operator with respect to the given probability measure $P$.

\section{Preliminaries}

Considering the following stochastic neutral-type memristive neural network, for $i=1,2, \ldots, n$,

$$
\begin{array}{r}
d\left[x_{i}(t)-\sum_{j=1}^{n} d_{i j} x_{j}(t-\tau(t))\right]=\left[-c_{i} x_{i}(t)\right. \\
+\sum_{j=1}^{n} a_{i j}\left(x_{i}(t)\right) f_{j}\left(x_{j}(t)\right)+\sum_{j=1}^{n} b_{i j}\left(x_{i}(t)\right)
\end{array}
$$

$$
\begin{aligned}
& \cdot g_{j}\left(x_{j}(t-\tau(t))\right. \\
& +\sum_{j=1}^{n} e_{i j}\left(x_{i}(t)\right) \int_{-\infty}^{0} h_{j}\left(x_{j}(t+\theta)\right) d \eta_{j}(\theta) \\
& \left.\left.+u_{i}(t)\right)\right] d t+\sum_{j=1}^{n} \sigma_{i j}\left(t, x_{j}(t), x_{j}(t\right. \\
& -\tau(t))) d \omega_{j}(t)
\end{aligned}
$$

with initial conditions $x_{i}(t)=\phi_{i}(t), t \in[-\tau, 0]$, where $x_{i}(t)$ is the voltage of the capacitor $\mathbf{C}_{i}, f_{j}\left(x_{j}(t)\right), g_{j}\left(x_{j}(t)\right)$, and $h_{j}\left(x_{j}(t)\right)$ represent the neuron activation functions of the $j$ th neuron at time $t, u_{i}(t) \in l_{\infty}$ is the external constant input of the $i$ th neuron at time $t, \omega_{j}$ is a standard Brownian motion defined on the complete probability space $(\Omega, F, P)$ with a natural filtration $\left\{F_{t}\right\}_{t \geq 0}$, and $\sigma_{i j}$ is a Borel measurable function. $C=\operatorname{diag}\left(c_{1}, c_{2}, \ldots, c_{n}\right)$ and $D=$ $\operatorname{diag}\left(d_{1}, d_{2}, \ldots, d_{n}\right)$ are self-feedback connection matrices, and $a_{i j}\left(x_{i}(t)\right), b_{i j}\left(x_{i}(t)\right)$, and $e_{i j}\left(x_{i}(t)\right)$ represent memristorbased weights,

$$
\begin{aligned}
& a_{i j}\left(x_{i}(t)\right)=\frac{\mathbf{W}_{(1) i j}}{\mathbf{C}_{i}} \times \operatorname{sign}_{i j}, \\
& b_{i j}\left(x_{i}(t)\right)=\frac{\mathbf{W}_{(2) i j}}{\mathbf{C}_{i}} \times \operatorname{sign}_{i j}, \\
& e_{i j}\left(x_{i}(t)\right)=\frac{\mathbf{W}_{(3) i j}}{\mathbf{C}_{i}} \times \operatorname{sign}_{i j}, \\
& \operatorname{sign}_{i j}= \begin{cases}1 & i \neq j, \\
-1 & i=j,\end{cases}
\end{aligned}
$$

where $\mathbf{W}_{(k) i j}$ denote the memductances of memristors $\mathbf{R}_{(k) i j}, k=1,2,3$. And $\int_{-\infty}^{0} h_{j}\left(x_{j}(t+\theta)\right) d \eta_{j}(\theta)$ is the LebesgueStieltjes integral and $\eta_{j}(\theta)$ is nonnegative function of bounded variation on $(-\infty, 0]$, which satisfies $\int_{-\infty}^{0} d \eta_{j}(\theta)=$ $l_{j}>0$. According to the pinched hysteretic loops of ideal memristors, let

$$
\begin{aligned}
& a_{i j}\left(x_{i}(t)\right)= \begin{cases}a^{\prime} & \left|x_{i}(t)\right| \leq 1, \\
a^{\prime \prime} & \left|x_{i}(t)\right|>1,\end{cases} \\
& b_{i j}\left(x_{i}(t)\right)= \begin{cases}b^{\prime} & \left|x_{i}(t)\right| \leq 1, \\
b^{\prime \prime} & \left|x_{i}(t)\right|>1,\end{cases} \\
& e_{i j}\left(x_{i}(t)\right)= \begin{cases}e^{\prime} & \left|x_{i}(t)\right| \leq 1, \\
e^{\prime \prime} & \left|x_{i}(t)\right|>1\end{cases}
\end{aligned}
$$

for $i=1,2, \ldots, n$, and $a^{\prime}, a^{\prime \prime}, b^{\prime}, b^{\prime \prime}, e^{\prime}$, and $e^{\prime \prime}$ are constants. Let $\underline{a}_{i j}=\min \left\{a^{\prime}, a^{\prime \prime}\right\}, \bar{a}_{i j}=\max \left\{a^{\prime}, a^{\prime \prime}\right\}, \underline{b}_{i j}=\min \left\{b^{\prime}, b^{\prime \prime}\right\}$, 
$\bar{b}_{i j}=\max \left\{b^{\prime}, b^{\prime \prime}\right\}, \underline{e}_{i j}=\min \left\{e^{\prime}, e^{\prime \prime}\right\}, \bar{e}_{i j}=\max \left\{e^{\prime}, e^{\prime \prime}\right\}$, for $i, j=1,2, \ldots, n$. By applying theory of differential inclusions and set-valued maps in system (1), it follows that

$$
\begin{aligned}
& d\left[x_{i}(t)-\sum_{j=1}^{n} d_{i j} x_{j}(t-\tau(t))\right] \in\left[-c_{i} x_{i}(t)\right. \\
& +\sum_{j=1}^{n} \operatorname{co}\left[\underline{a}_{i j}, \bar{a}_{i j}\right] f_{j}\left(x_{j}(t)\right)+\sum_{j=1}^{n} \operatorname{co}\left[\underline{b}_{i j}, \bar{b}_{i j}\right] \\
& \quad \cdot g_{j}\left(x_{j}(t-\tau(t))\right. \\
& \left.\left.+\sum_{j=1}^{n} \operatorname{co}\left[\underline{e}_{i j}, \bar{e}_{i j}\right] \int_{-\infty}^{0} h_{j}\left(x_{j}(t+\theta)\right) d \eta_{j}(\theta)+u_{i}(t)\right)\right] d t \\
& \quad+\sum_{j=1}^{n} \sigma_{i j}\left(t, x_{j}(t), x_{j}(t-\tau(t))\right) d \omega_{j}(t),
\end{aligned}
$$

with initial conditions $x_{i}(t)=\phi_{i}(t), t \in[-\tau, 0]$, for $i, j=$ $1,2, \ldots, n$. Using Filippovs Theorem in [24], there exist $\widehat{a}_{i j} \in$ $\left[\underline{a}_{i j}, \bar{a}_{i j}\right], \widehat{b}_{i j} \in\left[\underline{b}_{i j}, \bar{b}_{i j}\right]$, and $\hat{e}_{i j} \in\left[\underline{e}_{i j}, \bar{e}_{i j}\right]$ such that

$$
\begin{aligned}
& d\left[x_{i}(t)-\sum_{j=1}^{n} d_{i j} x_{j}(t-\tau(t))\right]=\left[-c_{i} x_{i}(t)\right. \\
& \quad+\sum_{j=1}^{n} \widehat{a}_{i j} f_{j}\left(x_{j}(t)\right)+\sum_{j=1}^{n} \widehat{b}_{i j} g_{j}\left(x_{j}(t-\tau(t))+\sum_{j=1}^{n} \widehat{e}_{i j}\right. \\
& \left.\left.\quad \cdot \int_{-\infty}^{0} h_{j}\left(x_{j}(t+\theta)\right) d \eta_{j}(\theta)+u_{i}(t)\right)\right] d t \\
& \quad+\sum_{j=1}^{n} \sigma_{i j}\left(t, x_{j}(t), x_{j}(t-\tau(t))\right) d \omega_{j}(t),
\end{aligned}
$$

with initial conditions $x_{i}(t)=\phi_{i}(t), t \in[-\tau, 0]$, for $i, j=$ $1,2, \ldots, n$.

To obtain the main results, we need the following hypotheses.

$\left(H_{1}\right) f_{j}, g_{j}$, and $h_{j}$ satisfy the following conditions:

$$
\begin{aligned}
& \left|f_{j}\left(s_{1}\right)-f_{j}\left(s_{2}\right)\right| \leq \alpha_{i}\left|s_{1}-s_{2}\right|, \\
& \left|g_{j}\left(s_{1}\right)-g_{j}\left(s_{2}\right)\right| \leq \beta_{i}\left|s_{1}-s_{2}\right|, \\
& \left|h_{j}\left(s_{1}\right)-h_{j}\left(s_{2}\right)\right| \leq \gamma_{i}\left|s_{1}-s_{2}\right|,
\end{aligned}
$$

where $\alpha_{i}, \beta_{i}$, and $\gamma_{i}$ are positive constants, $\forall s_{1}, s_{2} \in R$, $j=1,2, \ldots, n$.

$\left(H_{2}\right)$ For all $i, j=1,2, \ldots, n, \exists \mu_{i j}, v_{i j} \geq 0$, which satisfy

$$
\begin{aligned}
& \left|\sigma_{i j}\left(t, u_{1}, v_{1}\right)-\sigma_{i j}\left(t, u_{2}, v_{2}\right)\right|^{2} \\
& \quad \leq \mu_{i j}\left|u_{1}-u_{2}\right|^{2}+v_{i j}\left|v_{1}-v_{2}\right|^{2} .
\end{aligned}
$$

$\left(H_{3}\right) \tau(t)$ satisfies the following conditions:

$$
\begin{aligned}
& 0 \leq \tau(t) \leq \tau, \\
& 0 \leq \dot{\tau}(t) \leq \chi<1,
\end{aligned}
$$

where $\tau$ and $\pi$ are positive constants.

$\left(H_{4}\right) \forall \theta \in(-\infty, 0]$, there exists a positive constant $\beta_{0}$, which satisfies

$$
\int_{-\infty}^{0} e^{-\beta \theta} d \eta_{j}(\theta)=K<+\infty
$$

where $\beta \in\left[0, \beta_{0}\right)$.

$\left(H_{5}\right) D=\left(d_{i j}\right)_{n \times n}$ is a matrix, $\|\cdot\|$ represents the matrix norm which satisfies $\eta_{0}=\|D\| \in(0,1)$, where matrix norm is defined as $\|D\|=\sqrt{\lambda_{\max }\left(D D^{T}\right)}$, and $\lambda_{\max }(\cdot)$ means the maximum eigenvalue (spectral radius) of the matrix.

Definition 1 (see [25]). The trivial solution of system (1) is said to be mean-square exponentially input-to-state stable if for every $\phi \in L_{F_{0}}^{2}\left([-\tau, 0] ; R^{n}\right)$ and $u(t) \in l_{\infty}$ there exist scalars $\alpha_{0}>0, \beta_{0}>0$, and $\gamma_{0}>0$ such that the following inequality holds:

$$
E\left[|x(t ; \phi)|^{2}\right] \leq \alpha_{0} e^{-\beta_{0} t} E\left[\|\phi\|^{2}\right]+\gamma_{0}\|u\|_{\infty}^{2} .
$$

\section{Main Results}

In this section, the mean-square exponential input-to-state stability of the trivial solution for system (1) is addressed.

Theorem 2. Under $\left(H_{1}\right)-\left(H_{5}\right)$, the trivial solution of system (1) is mean-square exponentially input-to-state stable, if there exist positive constants $p_{i}, \delta_{i},(i=1, \ldots, n)$ such that the following conditions hold:

$$
\begin{aligned}
& A_{1 i}=-2 p_{i} c_{i}+p_{i}+\delta_{i}+\sum_{j=1}^{n}\left[p _ { i } \left(c_{i}\left|d_{i j}\right|+\alpha_{j}\left|\widehat{a}_{i j}\right|\right.\right. \\
& \left.+\beta_{j}\left|\widehat{b}_{i j}\right|+l_{i}\left|\widehat{e}_{i j}\right|\right)+p_{j}\left(\alpha_{i}\left|\widehat{a}_{j i}\right|+\mu_{i j}+\gamma_{i}\left|\widehat{e}_{i j}\right|\right. \\
& \left.\left.\cdot \int_{-\infty}^{0} e^{-\beta \theta} d \eta_{j}(\theta)\right)\right]+\sum_{j=1}^{n} \sum_{k=1}^{n} p_{j}\left|d_{j k}\right|\left[\alpha_{i}\left|\widehat{a}_{j k}\right|\right. \\
& \left.+\gamma_{i}\left|\widehat{e}_{j i}\right| \int_{-\infty}^{0} e^{-\beta \theta} d \eta_{j}(\theta)\right]<0, \\
& A_{2 i}=-(1-\chi) \delta_{i}+\sum_{j=1}^{n} p_{j}\left(c_{j}\left|d_{j i}\right|+\beta_{i}\left|\widehat{b}_{i j}\right|+\left|d_{j i}\right|\right. \\
& \left.+v_{j i}\right)+\sum_{j=1}^{n} \sum_{k=1}^{n} p_{i}\left[\left|d_{j i}\right|\right. \\
& \left.\quad \cdot\left(\alpha_{k}\left|\widehat{b}_{j k}\right|+\beta_{k}\left|\widehat{a}_{j k}\right|+l_{k}\left|\widehat{e}_{j k}\right|+\beta_{i}\left|\widehat{b}_{j i}\right|\left|d_{j k}\right|\right)\right]<0 .
\end{aligned}
$$


Proof. In order to obtain the mean-square exponential input-to-state stability, we consider the following LyapunovKrasovskii functional:

$$
V(t, x(t))=V_{1}(t, x(t))+V_{2}(t, x(t)),
$$

where

$$
\begin{aligned}
& V_{1}(t, x(t))=e^{\beta t} \sum_{i=1}^{n} p_{i}\left[x_{i}(t)-\sum_{j=1}^{n} d_{i j} x_{j}(t-\tau(t))\right]^{2}, \\
& V_{2}(t, x(t))=\int_{t-\tau(t)}^{t} e^{\beta s} \sum_{i=1}^{n} \delta_{i}\left|x_{i}(s)\right|^{2} d s \\
& +\sum_{i=1}^{n} \sum_{j=1}^{n} p_{i}\left|\widehat{e}_{i j}\right| \gamma_{j}\left(1+\sum_{k=1}^{n}\left|d_{i k}\right|\right) \\
& \quad \cdot \int_{-\infty}^{0} \int_{t+\theta}^{t} e^{\beta(s-\theta)}\left|x_{j}(s)\right|^{2} d s d \eta_{j}(\theta) .
\end{aligned}
$$

By Itô formula, it follows that

$$
d V(t, x(t))=L V(t, x(t)) d t+V_{x}(t, x(t)) \sigma(t) d t
$$

where $V_{x}(t, x(t))=\left(\partial V(t, x(t)) / \partial x_{1}, \ldots, \partial V(t, x(t)) / \partial x_{n}\right)$ and $L$ is the weak infinitesimal operator which satisfies

$$
\begin{aligned}
& L V_{1}(t, x(t)) \\
& =\beta e^{\beta t} \sum_{i=1}^{n} p_{i}\left[x_{i}(t)-\sum_{j=1}^{n} d_{i j} x_{j}(t-\tau(t))\right]^{2} \\
& +2 e^{\beta t} \sum_{i=1}^{n} p_{i}\left[x_{i}(t)-\sum_{j=1}^{n} d_{i j} x_{j}(t-\tau(t))\right] \\
& +\left[-c_{i} x_{i}(t)+\sum_{j=1}^{n} \widehat{a}_{i j} f_{j}\left(x_{j}(t)\right)\right. \\
& +\sum_{j=1}^{n} \widehat{b}_{i j} g_{j}\left(x_{j}(t-\tau(t))\right) \\
& \left.+\sum_{j=1}^{n} \widehat{e}_{i j} \int_{-\infty}^{0} h_{j}\left(x_{j}(t+\theta) d \eta_{j}(\theta)+u_{i}(t)\right)\right] \\
& +2 e^{\beta t} \sum_{i=1}^{n} p_{i} c_{i} x_{i}^{2}(t) \\
& +e^{\beta t} \sum_{i=1}^{n} \sum_{j=1}^{n} p_{i} \sigma_{i j}^{2}\left(t, x_{j}(t), x_{j}(t-\tau(t))\right) \leq 2 \beta e^{\beta t} \\
& +p_{i=1}^{n}\left\{x_{i}^{2}(t)+\eta_{0} x_{j}^{2}(t-\tau(t))\right]
\end{aligned}
$$

$$
\begin{aligned}
& +2 e^{\beta t} \sum_{i=1}^{n} \sum_{j=1}^{n} p_{i} c_{i} d_{i j} x_{j}(t-\tau(t)) x_{i}(t) \\
& +2 e^{\beta t} \sum_{i=1}^{n} \sum_{j=1}^{n} p_{i} \widehat{a}_{i j} f_{j}\left(x_{j}(t)\right) x_{i}(t) \\
& -2 e^{\beta t} \sum_{i=1}^{n} \sum_{j=1}^{n} p_{i} d_{i j} \widehat{a}_{i j} x_{j}(t-\tau(t)) \sum_{k=1}^{n} f_{k}\left(x_{k}(t)\right) \\
& +2 e^{\beta t} \sum_{i=1}^{n} \sum_{j=1}^{n} p_{i} \widehat{b}_{i j} g_{j}\left(x_{j}(t-\tau(t)) x_{i}(t)\right) \\
& -2 e^{\beta t} \sum_{i=1}^{n} \sum_{j=1}^{n} p_{i} d_{i j} x_{j}(t-\tau(t)) \\
& \cdot \sum_{k=1}^{n} \widehat{b}_{i k} g_{k}\left(x_{k}(t-\tau(t))\right)+2 e^{\beta t} \sum_{i=1}^{n} \sum_{j=1}^{n} p_{i} \widehat{e}_{i j} \\
& \cdot \int_{-\infty}^{0} h_{j}\left(x_{j}(t+\theta)\right) d \eta_{j}(\theta) x_{i}(t) \\
& +2 e^{\beta t} \sum_{i=1}^{n} \sum_{j=1}^{n} p_{i} d_{i j} x_{j}(t-\tau(t)) \sum_{k=1}^{n} \widehat{e}_{i k} \\
& \cdot \int_{-\infty}^{0} h_{k}\left(x_{k}(t+\theta)\right) d \eta_{k}(\theta) \\
& +2 e^{\beta t} \sum_{i=1}^{n} p_{i} x_{i}(t) u_{i}(t)+2 e^{\beta t} \sum_{i=1}^{n} p_{i} \\
& \cdot \sum_{j=1}^{n} d_{i j} x_{j}(t-\tau(t)) u_{i}(t) \\
& +e^{\beta t} \sum_{i=1}^{n} \sum_{j=1}^{n} p_{i} \sigma_{i j}^{2}\left(t, x_{j}(t), x_{j}(t-\tau(t))\right),
\end{aligned}
$$

where $D=\left(d_{i j}\right)_{n \times n}$ is a matrix which satisfies $\|D x\| \leq$ $\|D\|\|x\|=\eta_{0}\|x\|$, and under $\left(H_{1}\right)$ and $\left(H_{2}\right)$ we have

$$
\begin{aligned}
& L V_{1}(t, x(t)) \leq 2 \beta e^{\beta t} \max \left\{p_{i}\right\} \\
& \cdot \sum_{i=1}^{n}\left[x_{i}^{2}(t)+\eta_{0} x_{j}^{2}(t-\tau(t))\right]-2 e^{\beta t} \sum_{i=1}^{n} p_{i} c_{i} x_{i}^{2}(t) \\
& +2 e^{\beta t} \sum_{i=1}^{n} \sum_{j=1}^{n} p_{i} c_{i}\left|d_{i j}\right|\left|x_{j}(t-\tau(t))\right|\left|x_{i}(t)\right| \\
& +2 e^{\beta t} \sum_{i=1}^{n} \sum_{j=1}^{n} p_{i}\left|\widehat{a}_{i j}\right| \alpha_{j}\left|x_{j}(t)\right|\left|x_{i}(t)\right| \\
& +2 e^{\beta t} \sum_{i=1}^{n} \sum_{j=1}^{n} p_{i}\left|d_{i j}\right|\left|\widehat{a}_{i j}\right|\left|x_{j}(t-\tau(t))\right| \sum_{k=1}^{n} \alpha_{k}\left|x_{k}(t)\right|
\end{aligned}
$$




$$
\begin{aligned}
& +2 e^{\beta t} \sum_{i=1}^{n} \sum_{j=1}^{n} p_{i}\left|\widehat{b}_{i j}\right| \beta_{j}\left|x_{j}(t-\tau(t))\right|\left|x_{i}(t)\right| \\
& +2 e^{\beta t} \sum_{i=1}^{n} \sum_{j=1}^{n} p_{i}\left|d_{i j}\right|\left|x_{j}(t-\tau(t))\right| \\
& \cdot \sum_{k=1}^{n}\left|\widehat{b}_{i k}\right| \beta_{k}\left|x_{k}(t-\tau(t))\right|+2 e^{\beta t} \sum_{i=1}^{n} \sum_{j=1}^{n} p_{i}\left|\widehat{e}_{i j}\right| \\
& \cdot \int_{-\infty}^{0}\left|h_{j}\left(x_{j}(t+\theta)\right)\right|\left|x_{i}(t)\right| d \eta_{j}(\theta) \\
& +2 e^{\beta t} \sum_{i=1}^{n} \sum_{j=1}^{n} \sum_{k=1}^{n} p_{i}\left|d_{i j}\right|\left|\hat{e}_{i k}\right| \\
& \cdot \int_{-\infty}^{0}\left|h_{k}\left(x_{k}(t+\theta)\right)\right|\left|x_{j}(t-\tau(t))\right| d \eta_{k}(\theta) \\
& +2 e^{\beta t} \sum_{i=1}^{n} p_{i} x_{i}(t) u_{i}(t)+2 e^{\beta t} \sum_{i=1}^{n} p_{i} \\
& \cdot \sum_{j=1}^{n} d_{i j} x_{j}(t-\tau(t)) u_{i}(t)+e^{\beta t} \sum_{i=1}^{n} \sum_{j=1}^{n} p_{i} \mu_{i j} x_{j}^{2}(t) \\
& +v_{i j} x_{j}^{2}(t-\tau(t)) .
\end{aligned}
$$

By the inequality $a^{2}+b^{2} \geq 2 a b$, we can obtain

$$
\begin{aligned}
& L V_{1}(t, x(t)) \leq 2 \beta e^{\beta t} \max \left\{p_{i}\right\} \sum_{i=1}^{n}\left[x_{i}^{2}(t)+\eta_{0} x_{j}^{2}(t\right. \\
& -\tau(t))]-2 e^{\beta t} \sum_{i=1}^{n} p_{i} c_{i} x_{i}^{2}(t)+e^{\beta t} \sum_{i=1}^{n} \sum_{j=1}^{n} p_{i} c_{i}\left|d_{i j}\right| \\
& \cdot\left[x_{j}^{2}(t-\tau(t))+x_{i}^{2}(t)\right]+e^{\beta t} \sum_{i=1}^{n} \sum_{j=1}^{n} p_{i}\left|\widehat{a}_{i j}\right| \\
& \cdot \alpha_{j}\left[x_{j}^{2}(t)+x_{i}^{2}(t)\right]+e^{\beta t} \sum_{i=1}^{n} \sum_{j=1}^{n} \sum_{k=1}^{n} p_{i}\left|\widehat{a}_{i k}\right| \alpha_{k}\left|d_{i j}\right| \\
& \cdot\left[\left|x_{j}(t-\tau(t))\right|^{2}+\left|x_{k}(t)\right|^{2}\right]+e^{\beta t} \sum_{i=1}^{n} \sum_{j=1}^{n} p_{i}\left|\widehat{b}_{i j}\right| \\
& \cdot \beta_{j}\left[\left|x_{j}(t-\tau(t))\right|^{2}+\left|x_{i}(t)\right|^{2}\right] \\
& +e^{\beta t} \sum_{i=1}^{n} \sum_{j=1}^{n} \sum_{k=1}^{n} p_{i}\left|\widehat{b}_{i k}\right| \beta_{k}\left|d_{i j}\right| \\
& \cdot\left[\left|x_{j}(t-\tau(t))\right|^{2}+\left|x_{k}(t-\tau(t))\right|^{2}\right] \\
& +e^{\beta t} \sum_{i=1}^{n} \sum_{j=1}^{n} p_{i}\left|\widehat{e}_{i j}\right| \int_{-\infty}^{0}\left[\left|h_{j}\left(x_{j}(t+\theta)\right)\right|^{2}\right.
\end{aligned}
$$

$$
\left.+\left|x_{i}(t)\right|^{2}\right] d \eta_{j}(\theta)
$$$$
+e^{\beta t} \sum_{i=1}^{n} \sum_{j=1}^{n} \sum_{k=1}^{n} p_{i}\left|d_{i j}\right|\left|\widehat{e}_{i k}\right| \int_{-\infty}^{0}\left[\left|h_{k}\left(x_{k}(t+\theta)\right)\right|^{2}\right.
$$$$
\left.+\left|x_{j}(t-\tau(t))\right|^{2}\right] d \eta_{k}(\theta)+e^{\beta t} \sum_{i=1}^{n} p_{i}
$$$$
\cdot \sum_{j=1}^{n}\left|d_{i j}\right|\left[\left|x_{j}(t-\tau(t))\right|^{2}+\left|u_{i}(t)\right|^{2}\right]
$$$$
+e^{\beta t} \sum_{i=1}^{n} p_{i}\left[\left|x_{i}\right|^{2}+\left|u_{i}(t)\right|^{2}\right]+e^{\beta t} \sum_{i=1}^{n} \sum_{j=1}^{n} p_{i} \mu_{i j} x_{j}^{2}(t)
$$$$
+v_{i j} x_{j}^{2}(t-\tau(t)) \leq 2 \beta e^{\beta t} \max \left\{p_{i}\right\} \sum_{i=1}^{n}\left[x_{i}^{2}(t)\right.
$$$$
\left.+\eta_{0} x_{j}^{2}(t-\tau(t))\right]-2 e^{\beta t} \sum_{i=1}^{n} p_{i} c_{i} x_{i}^{2}(t)
$$$$
+e^{\beta t} \sum_{i=1}^{n} \sum_{j=1}^{n} p_{i} c_{i}\left|d_{i j}\right|\left[x_{j}^{2}(t-\tau(t))+x_{i}^{2}(t)\right]
$$$$
+e^{\beta t} \sum_{i=1}^{n} \sum_{j=1}^{n} p_{i}\left|\widehat{a}_{i j}\right| \alpha_{j}\left[x_{j}^{2}(t)+x_{i}^{2}(t)\right]
$$$$
+e^{\beta t} \sum_{i=1}^{n} \sum_{j=1}^{n} \sum_{k=1}^{n} p_{i}\left|\widehat{a}_{i k}\right| \alpha_{k}\left|d_{i j}\right|
$$

$\cdot\left[\left|x_{j}(t-\tau(t))\right|^{2}+\left|x_{k}(t)\right|^{2}\right]+e^{\beta t} \sum_{i=1}^{n} \sum_{j=1}^{n} p_{i}\left|\widehat{b}_{i j}\right|$

$\cdot \beta_{j}\left[\left|x_{j}(t-\tau(t))\right|^{2}+\left|x_{i}(t)\right|^{2}\right]$

$$
+e^{\beta t} \sum_{i=1}^{n} \sum_{j=1}^{n} \sum_{k=1}^{n} p_{i}\left|\widehat{b}_{i k}\right| \beta_{k}\left|d_{i j}\right|
$$$$
\cdot\left[\left|x_{j}(t-\tau(t))\right|^{2}+\left|x_{k}(t-\tau(t))\right|^{2}\right]
$$$$
+e^{\beta t} \sum_{i=1}^{n} \sum_{j=1}^{n} p_{i}\left|\widehat{e}_{i j}\right|\left[\int_{-\infty}^{0} \gamma_{j}\left|x_{j}(t+\theta)\right|^{2} d \eta_{j}(\theta)\right.
$$$$
\left.+l_{j}\left|x_{i}(t)\right|^{2}\right]+e^{\beta t} \sum_{i=1}^{n} \sum_{j=1}^{n} \sum_{k=1}^{n} p_{i}\left|d_{i j}\right|\left|\widehat{e}_{i k}\right|
$$$$
\cdot\left[\int_{-\infty}^{0} \gamma_{k}\left|x_{k}(t+\theta)\right|^{2} d \eta_{k}(\theta)+l_{k}\left|x_{j}(t-\tau(t))\right|^{2}\right]
$$$$
+e^{\beta t} \sum_{i=1}^{n} p_{i} \sum_{j=1}^{n}\left|d_{i j}\right|\left[\left|x_{j}(t-\tau(t))\right|^{2}+\left|u_{i}(t)\right|^{2}\right]
$$$$
+e^{\beta t} \sum_{i=1}^{n} p_{i}\left[\left|x_{i}\right|^{2}+\left|u_{i}(t)\right|^{2}\right]+e^{\beta t} \sum_{i=1}^{n} \sum_{j=1}^{n} p_{i}\left[\mu_{i j} x_{j}^{2}(t)\right.
$$$$
\left.+v_{i j} x_{j}^{2}(t-\tau(t))\right] \text {, }
$$

$L V_{2}(t, x(t))=e^{\beta t} \sum_{i=1}^{n} \delta_{i}\left|x_{i}(t)\right|^{2}-(1-\dot{\tau}(t)) e^{\beta(t-\tau(t))}$ 


$$
\begin{aligned}
& \cdot \sum_{i=1}^{n} \delta_{i}\left|x_{i}(t-\tau(t))\right|^{2}+\sum_{i=1}^{n} \sum_{j=1}^{n} p_{i}\left|\widehat{e}_{i j}\right| \gamma_{j}(1 \\
& \left.+\sum_{k=1}^{n}\left|d_{i k}\right|\right) \int_{-\infty}^{0} e^{\beta(t-\theta)}\left|x_{j}(t)\right|^{2} d \eta_{j}(\theta) \\
& -\sum_{i=1}^{n} \sum_{j=1}^{n} p_{i}\left|\widehat{e}_{i j}\right| \gamma_{j}\left(1+\sum_{k=1}^{n}\left|d_{i k}\right|\right) \int_{-\infty}^{0} e^{\beta(t)} \mid x_{j}(t
\end{aligned}
$$$$
+\theta)\left.\right|^{2} d \eta_{j}(\theta) \leq e^{\beta t} \sum_{i=1}^{n} \delta_{i}\left|x_{i}(t)\right|^{2}
$$$$
-(1-\chi) e^{\beta(t-\tau)} \sum_{i=1}^{n} \delta_{i}\left|x_{i}(t-\tau(t))\right|^{2}
$$

By condition (12), there exists a sufficiently small constant $\beta>$ 0 such that

$$
\begin{aligned}
& L V(t, x(t)) \leq e^{\beta t} \sum_{i=1}^{n}\left\{2 \beta \max \left\{p_{i}\right\}-2 p_{i} c_{i}+p_{i}+\delta_{i}\right. \\
& \quad+\sum_{j=1}^{n}\left[p_{i}\left(c_{i}\left|d_{i j}\right|+\alpha_{j}\left|\widehat{a}_{i j}\right|+\beta_{j}\left|\widehat{b}_{i j}\right|+l_{i}\left|\widehat{e}_{i j}\right|\right)+p_{j}\left(\alpha_{i}\left|\widehat{a}_{j i}\right|+\mu_{i j}+\gamma_{i}\left|\widehat{e}_{i j}\right| \int_{-\infty}^{0} e^{-\beta \theta} d \eta_{i}(\theta)\right)\right] \\
& \left.+\sum_{j=1}^{n} \sum_{k=1}^{n} p_{j}\left|d_{j k}\right|\left[\alpha_{i}\left|\widehat{a}_{j k}\right|+\gamma_{i}\left|\widehat{e}_{j i}\right| \int_{-\infty}^{0} e^{-\beta \theta} d \eta_{i}(\theta)\right]\right\}\left|x_{i}(t)\right|^{2}+e^{\beta(t)} \sum_{i=1}^{n}\left\{2 \beta \max \left\{p_{i}\right\} \eta_{0}-(1-\chi) e^{-\beta \tau} \delta_{i}\right. \\
& \left.+\sum_{j=1}^{n} p_{j}\left(c_{j}\left|d_{j i}\right|+\beta_{i}\left|\widehat{b}_{i j}\right|+\left|d_{j i}\right|+v_{j i}\right)+\sum_{j=1}^{n} \sum_{k=1}^{n} p_{i}\left[\left|d_{j i}\right|\left(\alpha_{k}\left|\widehat{b}_{j k}\right|+\beta_{k}\left|\widehat{a}_{j k}\right|+l_{k}\left|\widehat{e}_{j k}\right|+\beta_{i}\left|\widehat{b}_{j i}\right|\left|d_{j k}\right|\right)\right]\right\}\left|x_{i}(t-\tau(t))\right|^{2} \\
& +e^{\beta t} \sum_{i=1}^{n} p_{i}\left(1+\sum_{j=1}^{n}\left|d_{i j}\right|\right)\left|u_{i}(t)\right|^{2} \leq e^{\beta t} \sum_{i=1}^{n} p_{i}\left(1+\sum_{j=1}^{n}\left|d_{i j}\right|\right)\left|u_{i}(t)\right|^{2} .
\end{aligned}
$$

Define $\tau_{k}:=\inf \{s \geq 0:|x(s) \geq k|\}$ as a stopping time; we have

$$
\begin{aligned}
& E V\left(t \wedge \tau_{k}, x\left(t \wedge \tau_{k}\right)\right) \\
& \quad=E V(0, x(0))+E\left[\int_{0}^{t \wedge \tau_{k}} V(s, x(s)) d s\right] .
\end{aligned}
$$

\section{Note that}

$$
\begin{aligned}
& E V\left(t \wedge \tau_{k}, x\left(t \wedge \tau_{k}\right)\right) \\
& \leq E V(0, x(0))
\end{aligned}
$$

$$
+\left(e^{\beta t}-1\right) \sum_{i=1}^{n} p_{i}\left(1+\sum_{j=1}^{n}\left|d_{i j}\right|\right)\left|u_{i}(t)\right|^{2},
$$

letting $k \rightarrow \infty$

$E V(0, x(0))$

$$
\begin{aligned}
& \leq\left[2 \max _{1 \leq i \leq n}\left\{p_{i}\right\}\left(1+\eta_{0}^{2}\right)+\max _{1 \leq i \leq n}\left\{\delta_{i}\right\} \tau+\max _{1 \leq i \leq n}\left\{\sum_{j=1}^{n} p_{j}\left|\widehat{e}_{j i}\right| \gamma_{i} \frac{\int_{-\infty}^{0}\left(e^{-\beta \theta}-1\right) d \eta_{j}(\theta)}{\beta}\left(1+\sum_{k=1}^{n}\left|d_{j k}\right|\right)\right\}\right] E\|\phi\|^{2} \\
& =K_{0} E\|\phi\|^{2} .
\end{aligned}
$$

Then we have

$$
E V(t, x(t)) \leq K_{0} E\|\phi\|^{2}+K_{1} e^{\beta t}\|u\|_{\infty}^{2},
$$

where $K_{1}=\sum_{i=1}^{n} p_{i}\left(1+\sum_{i=1}^{n}\left|d_{i j}\right|\right)$. By the inequalities $(m+$ $n)^{2} \leq(1+\varepsilon) m^{2}+(1+1 / \varepsilon) n^{2}, \varepsilon>0$, it follows that 


$$
\begin{aligned}
& \min _{1 \leq j \leq n}\left\{p_{j}\right\} E\left[e^{\beta t}|x(t)|^{2}\right]=\min _{1 \leq j \leq n}\left\{p_{j}\right\} E\left[e^{\beta t}(\mid(t)\right. \\
& \left.\left.-D x(t-\tau(t))+\left.D x(t-\tau(t))\right|^{2}\right)\right] \leq \min _{1 \leq j \leq n}\left\{p_{j}\right\} \\
& \cdot e^{\beta t} E\left[( 1 + \varepsilon ) \left(|x(t)-D x(t-\tau(t))|^{2}\right.\right. \\
& \left.\left.+\left(1+\frac{1}{\varepsilon}\right)|D x(t-\tau(t))|^{2}\right)\right] \leq(1+\varepsilon) E(t, x(t)) \\
& +\min _{1 \leq j \leq n}\left\{p_{j}\right\} \eta_{0}^{2}\left(1+\frac{1}{\varepsilon}\right) E\left[e^{\beta t}|x(t-\tau(t))|^{2}\right] \leq(1 \\
& +\varepsilon)\left(K_{0} E\|\phi\|^{2}+K_{1} e^{\beta t}\|u\|_{\infty}^{2}\right)+\min _{1 \leq j \leq n}\left\{p_{j}\right\} \eta_{0}^{2}(1 \\
& \left.+\frac{1}{\varepsilon}\right) E\left[e^{\beta t}|x(t-\tau(t))|^{2}\right] .
\end{aligned}
$$

We can find a large enough constant $\varepsilon$ and a sufficiently small constant $\beta$ such that $\eta_{0}^{2} e^{\beta \tau}(1+1 / \varepsilon)<1$, so

$$
\begin{aligned}
\sup _{-\tau \leq s \leq t} & \min _{1 \leq j \leq n}\left\{p_{j}\right\} E\left[e^{\beta s}|x(s)|^{2}\right] \\
\leq & \min _{1 \leq j \leq n}\left\{p_{j}\right\} E\|\phi\|^{2}+\sup _{0 \leq s \leq t} \min _{1 \leq j \leq n}\left\{p_{j}\right\} E\left[e^{\beta s}|x(s)|^{2}\right] \\
\leq & {\left[\min _{1 \leq j \leq n}\left\{p_{j}\right\}+(1+\varepsilon) K_{0}\right] E\|\phi\|^{2} } \\
& +(1+\varepsilon)\left(K_{1} e^{\beta t}\|u\|_{\infty}^{2}\right) \\
& +\sup _{-\tau \leq s \leq t} \min _{1 \leq j \leq n}\left\{p_{j}\right\} \eta_{0}^{2}\left(1+\frac{1}{\varepsilon}\right) e^{\beta \tau} E\left[e^{\beta s}|x(s)|^{2}\right] ;
\end{aligned}
$$

that is,

$$
\sup _{-\tau \leq s \leq t} E\left[e^{\beta s}|x(s)|^{2}\right] \leq \alpha E\|\phi\|^{2}+\gamma e^{\beta t}\|u\|_{\infty}^{2},
$$

where

$$
\begin{aligned}
& \alpha=\frac{\min _{1 \leq j \leq n}\left\{p_{j}\right\}+(1+\varepsilon) K_{0}}{\min _{1 \leq j \leq n}\left\{p_{j}\right\}\left(1-\eta_{0}^{2}(1+1 / \varepsilon) e^{\beta \tau}\right)}, \\
& \gamma=\frac{(1+\varepsilon) K_{1}}{\min _{1 \leq j \leq n}\left\{p_{j}\right\}\left(1-\eta_{0}^{2}(1+1 / \varepsilon) e^{\beta \tau}\right)} .
\end{aligned}
$$

Furthermore,

$$
E\left[|x(t)|^{2}\right] \leq \alpha e^{-\beta t} E\|\phi\|^{2}+\gamma\|u\|_{\infty}^{2} .
$$

Hence, the desired assertion is derived.

Theorem 3. Under $\left(H_{1}\right)-\left(H_{5}\right)$, if conditions of (12) are satisfied, the trivial solution of system (1) with $u_{i}(t)=0$ is meansquare exponentially stable.
Moreover, when we remove the S-type distributed delay system (1) becomes the following system:

$$
\begin{aligned}
& d\left[x_{i}(t)-\sum_{j=1}^{n} d_{i j} x_{j}(t-\tau(t))\right]=\left[-c_{i} x_{i}(t)\right. \\
& +\sum_{j=1}^{n} a_{i j}\left(x_{i}(t)\right) f_{j}\left(x_{j}(t)\right) \\
& \left.+\sum_{j=1}^{n} b_{i j}\left(x_{i}(t)\right) g_{j}\left(x_{j}(t-\tau(t))+u_{i}(t)\right)\right] d t \\
& +\sum_{j=1}^{n} \sigma_{i j}\left(t, x_{j}(t), x_{j}(t-\tau(t))\right) d \omega_{j}(t) .
\end{aligned}
$$

Corollary 4. Under $\left(\mathrm{H}_{1}\right)-\left(\mathrm{H}_{3}\right)$ and $\left(\mathrm{H}_{5}\right)$, the trivial solution of system (29) is mean-square exponentially input-to-state stable, if there exist positive constants $p_{i}, \delta_{i},(i=1, \ldots, n)$ such that the following conditions hold:

$$
\begin{aligned}
& A_{1 i}=-2 p_{i} c_{i}+p_{i}+\delta_{i}+\sum_{j=1}^{n}\left[p _ { i } \left(c_{i}\left|d_{i j}\right|+\alpha_{j}\left|\widehat{a}_{i j}\right|\right.\right. \\
& \left.\left.\quad+\beta_{j}\left|\widehat{b}_{i j}\right|\right)+p_{j}\left(\alpha_{i}\left|\widehat{a}_{j i}\right|+\mu_{i j}\right)\right]+\sum_{j=1}^{n} \sum_{k=1}^{n} p_{j}\left|d_{j k}\right| \\
& \quad \cdot \alpha_{i}\left|\widehat{a}_{j k}\right|<0, \\
& A_{2 i}=-(1-\chi) \delta_{i}+\sum_{j=1}^{n} p_{j}\left(c_{j}\left|d_{j i}\right|+\beta_{i}\left|\widehat{b}_{i j}\right|+\left|d_{j i}\right|\right. \\
& \left.\quad+v_{j i}\right)+\sum_{j=1}^{n} \sum_{k=1}^{n} p_{i}\left[\left|d_{j i}\right|\right. \\
& \left.\quad \cdot\left(\alpha_{k}\left|\widehat{b}_{j k}\right|+\beta_{k}\left|\widehat{a}_{j k}\right|+\beta_{i}\left|\widehat{b}_{j i}\right|\left|d_{j k}\right|\right)\right]<0 .
\end{aligned}
$$

Corollary 5. Under $\left(H_{1}\right)-\left(H_{3}\right)$ and $\left(H_{5}\right)$, if conditions of (30) are satisfied, the trivial solution of system (29) with $u_{i}(t)=0$ is mean-square exponentially stable.

Remark 6. In particular, when we remove the neutral terms, system (29) becomes the system in [23]; from [23] we can see that the trivial solution of system is mean-square exponentially input-to-state stable and the trivial solution of system with $u_{i}(t)=0$ is mean-square exponentially stable in the certain condition. So we can say that our model is the extension of model in [23].

Remark 7. In fact, let

$$
\eta_{j}(\theta)=\left\{\begin{array}{ll}
-1 & \theta \leq-\tau_{j}, \\
0 & -\tau_{j}<\theta \leq 0,
\end{array} \quad j=1,2, \ldots, n,\right.
$$

where $\eta_{j}(\theta)$ is nonnegative function of bounded variation on $(-\infty, 0]$ and S-type distributed delays terms become 
$\sum_{j=1}^{n} e_{i j}\left(x_{i}(t)\right) h_{j}\left(x_{j}\left(t-\tau_{j}\right)\right)$, so system (1) contains the system in [25]. In addition, suppose that $d \eta_{j}(\theta)=k(\theta) d \theta$; we have that S-type distributed delays terms become the generally distributed delays $\sum_{j=1}^{n} e_{i j}\left(x_{i}(t)\right) \int_{-\infty}^{0} k(\theta) h_{j}\left(x_{j}(t+\theta)\right) d \theta$, so our system contains the recent work of [26]. It shows that this paper is more general than the existing articles.

Remark 8. On the achievements of $[23,25]$, this paper discusses a class of more general neural network systems through introducing many factors such as neutral terms, Stype distributed delays, and stochastic perturbations and analyzes the mean-square exponential input-to-state stability of the given neutral stochastic system by utilizing the LyapunovKrasovskii functional method, stochastic analysis techniques, and Itô formula. The considered Lyapunov-Krasovskii functional in our paper is more complex comparing with those in $[23,25]$ since it covers neutral terms and double integrals. Therefore, our theoretical results can be seen as an extension in $[23,25]$. In addition, our results are computationally efficient as the sufficient conditions can be easily checked without using linear matrix inequality toolbox.

\section{Numerical Simulation}

In this section, a numerical example is given to illustrate the correctness of our conclusions. Consider a two-dimensional system with $f_{i}(x)=g_{i}(x)=0.1 \cos x, \tau(t)=0.4+0.1 \cos t$, $h_{i}(x)=0.3 x, \eta_{i}(\theta)=e^{\theta}, \theta \in(-\infty, 0], u_{1}(t)=0.5 \cos x(t)$, and $u_{2}(t)=0.2 \cos x(t)$ :

$$
\begin{aligned}
\left(\sigma_{i j}\right)_{2 \times 2} & =\left(\begin{array}{cc}
0.8 x_{1}(t) & 0.2 x_{2}(t-\tau(t)) \\
0.2 x_{1}(t-\tau(t)) & 0.7 x_{2}(t)
\end{array}\right) \\
\left(d_{i j}\right)_{2 \times 2} & =\left(\begin{array}{cc}
0.2 & 0 \\
0 & 0.3
\end{array}\right), \\
\left(a_{i j}\right)_{2 \times 2} & =\left(\begin{array}{ll}
a_{11}\left(x_{1}\right) & a_{12}\left(x_{1}\right) \\
a_{21}\left(x_{2}\right) & a_{22}\left(x_{2}\right)
\end{array}\right) \\
\left(b_{i j}\right)_{2 \times 2} & =\left(\begin{array}{ll}
b_{11}\left(x_{1}\right) & b_{12}\left(x_{1}\right) \\
b_{21}\left(x_{2}\right) & b_{22}\left(x_{2}\right)
\end{array}\right), \\
\left(e_{i j}\right)_{2 \times 2} & =\left(\begin{array}{ll}
e_{11}\left(x_{1}\right) & e_{12}\left(x_{1}\right) \\
e_{21}\left(x_{2}\right) & e_{22}\left(x_{2}\right)
\end{array}\right), \\
a_{1 j}\left(x_{1}(t)\right) & =\left\{\begin{array}{ll}
-0.1 & \left|x_{1}(t)\right| \leq 1, \\
0.1 & \left|x_{1}(t)\right|>1,
\end{array} \quad j=1,2 ;\right. \\
a_{2 j}\left(x_{2}(t)\right) & =\left\{\begin{array}{ll}
-0.1 & \left|x_{2}(t)\right| \leq 1, \\
0.1 & \left|x_{2}(t)\right|>1,
\end{array} \quad j=1,2 ;\right.
\end{aligned}
$$

$$
\begin{aligned}
& b_{2 j}\left(x_{2}(t)\right)=\left\{\begin{array}{ll}
-0.01 & \left|x_{2}(t)\right| \leq 1, \\
0.01 & \left|x_{2}(t)\right|>1,
\end{array} \quad j=1,2 ;\right. \\
& e_{11}\left(x_{1}(t)\right)= \begin{cases}-2 & \left|x_{1}(t)\right| \leq 1, \\
0.5 & \left|x_{1}(t)\right|>1\end{cases} \\
& e_{12}\left(x_{1}(t)\right)= \begin{cases}-0.1 & \left|x_{1}(t)\right| \leq 1, \\
0.1 & \left|x_{1}(t)\right|>1,\end{cases} \\
& e_{21}\left(x_{2}(t)\right)= \begin{cases}-0.1 & \left|x_{2}(t)\right| \leq 1, \\
0.1 & \left|x_{2}(t)\right|>1,\end{cases} \\
& e_{22}\left(x_{2}(t)\right)= \begin{cases}-2 & \left|x_{2}(t)\right| \leq 1 \\
1 & \left|x_{2}(t)\right|>1 .\end{cases}
\end{aligned}
$$

Take $p_{1}=0.125, p_{2}=0.095, c_{1}=2.7, c_{2}=3.18, \delta_{1}=\delta_{2}=$ $0.2, \tau=0.5, \chi=0.001, l_{1}=l_{2}=1, K=0.1, \alpha_{1}=\alpha_{2}=0.1$, $\beta_{1}=\beta_{2}=0.1$, and $\gamma_{1}=\gamma_{2}=0.3$, which satisfy $\left(H_{1}\right)$ to $\left(H_{5}\right)$. Then, it is easy to check following conditions:

$$
\begin{aligned}
& A_{11}=-2 p_{1} c_{1}+p_{1}+\delta_{1}+\sum_{j=1}^{2}\left[p _ { 1 } \left(c_{1}\left|d_{1 j}\right|+\alpha_{j}\left|\widehat{a}_{1 j}\right|\right.\right. \\
& \left.+\beta_{j}\left|\widehat{b}_{1 j}\right|+l_{1}\left|\widehat{e}_{1 j}\right|\right)+p_{j}\left(\alpha_{1}\left|\widehat{a}_{j 1}\right|+\mu_{1 j}+\gamma_{1}\left|\widehat{e}_{1 j}\right|\right. \\
& \cdot K)]+\sum_{j=1}^{2} \sum_{k=1}^{2} p_{j}\left|d_{j k}\right|\left[\alpha_{1}\left|\widehat{a}_{j k}\right|+\gamma_{1}\left|\widehat{e}_{j 1}\right| K\right] \\
& =-0.0479<0 \text {, } \\
& A_{12}=-2 p_{2} c_{2}+p_{2}+\delta_{2}+\sum_{j=1}^{2}\left[p _ { 2 } \left(c_{2}\left|d_{2 j}\right|+\alpha_{j}\left|\widehat{a}_{2 j}\right|\right.\right. \\
& \left.+\beta_{j}\left|\widehat{b}_{2 j}\right|+l_{2}\left|\widehat{e}_{2 j}\right|\right)+p_{j}\left(\alpha_{2}\left|\widehat{a}_{j 2}\right|+\mu_{2 j}+\gamma_{2}\left|\widehat{e}_{2 j}\right|\right. \\
& \cdot K)]+\sum_{j=1}^{2} \sum_{k=1}^{2} p_{j}\left|d_{j k}\right|\left[\alpha_{2}\left|\widehat{a}_{j k}\right|+\gamma_{2}\left|\widehat{e}_{j 2}\right| K\right] \\
& =-0.0548<0 \text {, } \\
& A_{21}=-(1-\chi) \delta_{1}+\sum_{j=1}^{2} p_{j}\left(c_{j}\left|d_{j 1}\right|+\beta_{1}\left|\widehat{b}_{1 j}\right|+\left|d_{j 1}\right|\right. \\
& \left.+v_{j 1}\right)+\sum_{j=1}^{2} \sum_{k=1}^{2} p_{1}\left[\left|d_{j 1}\right|\right. \\
& \left.\cdot\left(\alpha_{k}\left|\widehat{b}_{j k}\right|+\beta_{k}\left|\widehat{a}_{j k}\right|+l_{k}\left|\widehat{e}_{j k}\right|+\beta_{1}\left|\widehat{b}_{j 1}\right|\left|d_{j k}\right|\right)\right] \\
& =-0.0478<0 \text {, }
\end{aligned}
$$



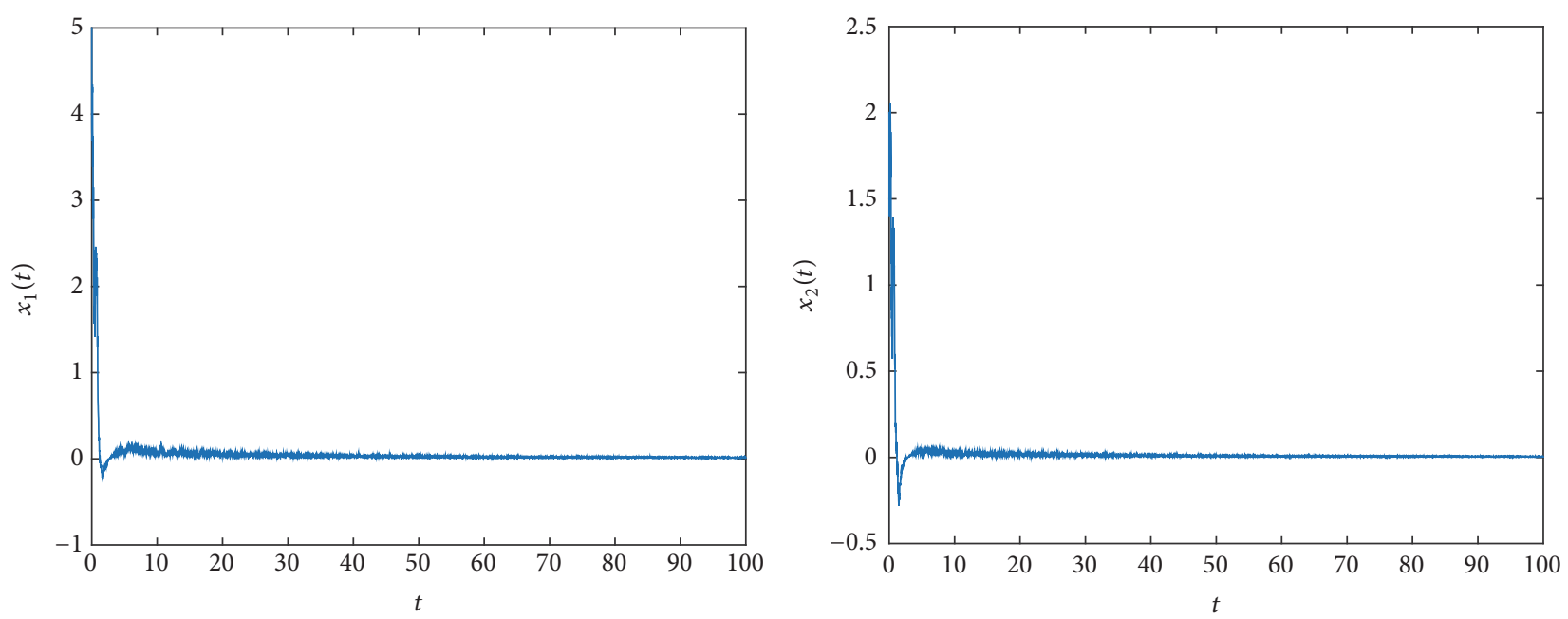

Figure 1: Transient response of variables $x(t)$.
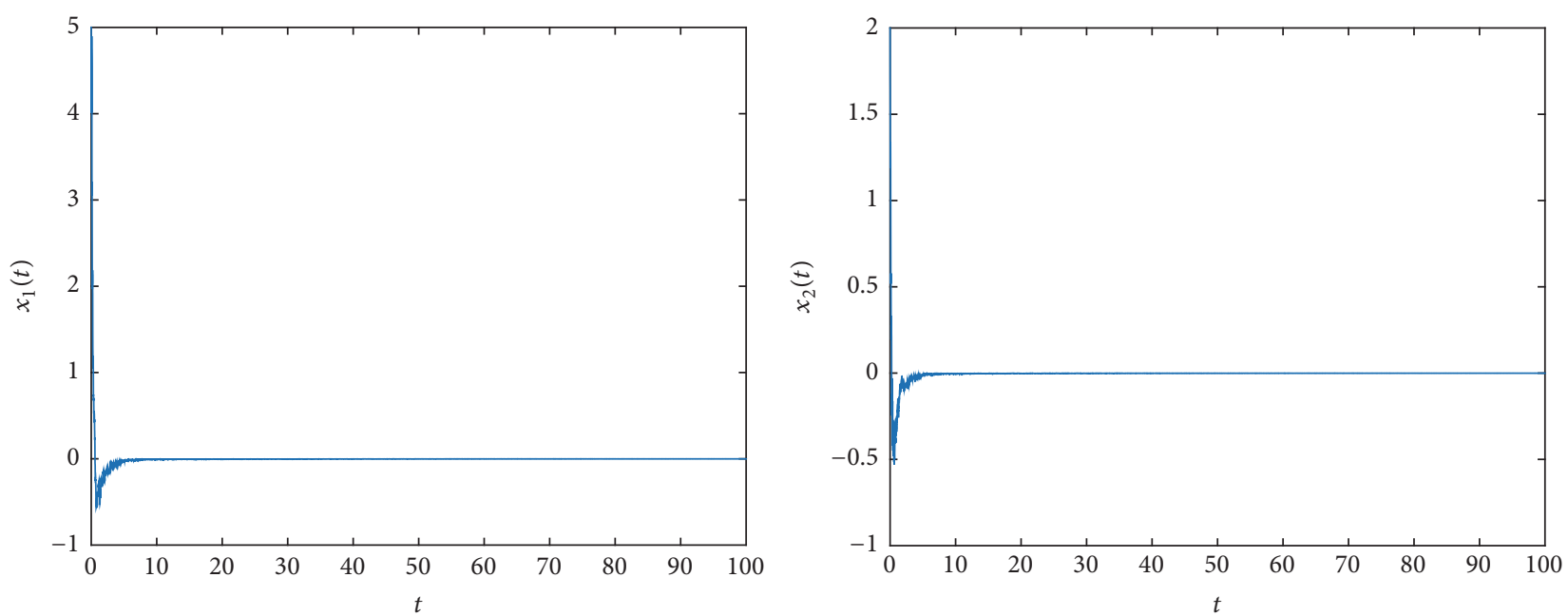

Figure 2: Transient response of variables $x(t)$ with $u(t)=0$.

$$
\begin{aligned}
& A_{22}=-(1-\chi) \delta_{2}+\sum_{j=1}^{2} p_{j}\left(c_{j}\left|d_{j 2}\right|+\beta_{2}\left|\widehat{b}_{2 j}\right|+\left|d_{j 2}\right|\right. \\
& \left.\quad+v_{j 2}\right)+\sum_{j=1}^{2} \sum_{k=1}^{2} p_{2}\left[\left|d_{j 2}\right|\right. \\
& \left.\quad \cdot\left(\alpha_{k}\left|\widehat{b}_{j k}\right|+\beta_{k}\left|\widehat{a}_{j k}\right|+l_{k}\left|\widehat{e}_{j k}\right|+\beta_{2}\left|\widehat{b}_{j 2}\right|\left|d_{j k}\right|\right)\right] \\
& \quad=-0.0150<0 .
\end{aligned}
$$

So the conditions of Theorem 2 are satisfied, and we can obtain that the trivial solution of system (1) is mean-square exponentially input-to-state stable with initial values $x(s)=$ $\phi(0)$; see Figure 1. When $u_{1}(t)=u_{2}(t)=0$ the trivial solution of system (1) is mean-square exponentially stable; see Figure 2.

\section{Conclusions and Discussion}

By stochastic analysis theory and Itô formula, mean-square exponential input-to-stability of a class of stochastic neutraltype memristive neural networks is studied. The correctness of our conclusions has been illustrated by a numerical example. In the current papers, there are few studies on stochastic neutral-type memristive neural networks with time-varying delay and S-type distributed delays. Furthermore, in this paper we discuss mean-square exponential input-to-stability of system (1); one may continue to discuss synchronization and passivity as well as some other complex dynamical behaviors on (1).

\section{Competing Interests}

The authors declare that they have no competing interests. 


\section{Acknowledgments}

The authors thank National Natural Science Foundation of China (no. 61563033, 11563005, and 11501281), Natural Science Foundation of Jiangxi Province (nos. 20122BAB201002 and 20151BAB212011), and Innovation Fund Designated for Graduate Students of Nanchang University (no. cx2015089) for their financial support.

\section{References}

[1] S. Ding and Z. Wang, "Stochastic exponential synchronization control of mem-ristive neural networks with multiple timevarying delays," Neurocomputing, vol. 162, pp. 16-25, 2015.

[2] W. Wang, L. Li, H. Peng, J. Xiao, and Y. Yang, "Synchronization control of memristor-based recurrent neural networks with perturbations," Neural Networks, vol. 53, pp. 8-14, 2014.

[3] H. Wang, Y. Yu, and G. Wen, "Stability analysis of fractionalorder Hopfield neural networks with time delays," Neural Networks, vol. 55, pp. 98-109, 2014.

[4] X. Zeng, Z. Xiong, and C. Wang, "Hopf bifurcation for neutraltype neural network model with two delays," Applied Mathematics and Computation, vol. 282, pp. 17-31, 2016.

[5] A. Wu and Z. Zeng, "Improved conditions for global exponential stability of a general class of memristive neural networks," Communications in Nonlinear Science and Numerical Simulation, vol. 20, no. 3, pp. 975-985, 2015.

[6] Y. Wan and J. Cao, "Periodicity and synchronization of coupled memristive neural networks with supremums," Neurocomputing, vol. 159, no. 1, pp. 137-143, 2015.

[7] G. Zhang, J. Hu, and Y. Shen, "Exponential lag synchronization for delayed memristive recurrent neural networks," Neurocomputing, vol. 154, pp. 86-93, 2015.

[8] J. Xiao, S. Zhong, and Y. Li, "New passivity criteria for memristive uncertain neural networks with leakage and time-varying delays," ISA Transactions, vol. 59, pp. 133-148, 2015.

[9] Y. Xin, Y. Li, Z. Cheng, and H. Xia, "Global exponential stability for switched memristive neural networks with time-varying delays," Neural Networks, vol. 80, pp. 34-42, 2016.

[10] Y. Liu, C. Li, T. Huang, and X. Wang, "Robust adaptive lag synchronization of uncertain fuzzy memristive neural networks with time-varying delays," Neurocomputing, vol. 190, pp. 188196, 2016.

[11] X. Han, H. Wu, and B. Fang, "Adaptive exponential synchronization of memristive neural networks with mixed timevarying delays," Neurocomputing, vol. 201, pp. 40-50, 2016.

[12] L. Wang and D. Xu, "Global asymptotic stability of bidirectional associative memory neural networks with S-type distributed delays," International Journal of Systems Science, vol. 33, no. 11, pp. 869-877, 2002.

[13] W. Han, Y. Kao, and L. Wang, "Global exponential robust stability of static interval neural networks with S-type distributed delays," Journal of the Franklin Institute, vol. 348, no. 8, pp. 20722081, 2011.

[14] Z. Huang, X. Li, S. Mohamad, and Z. Lu, "Robust stability analysis of static neural network with S-type distributed delays," Applied Mathematical Modelling, vol. 33, no. 2, pp. 760-769, 2009.

[15] G. Bao and Z. Zeng, "Global asymptotical stability analysis for a kind of discrete-time recurrent neural network with discontinuous activation functions," Neurocomputing, vol. 193, pp. 242-249, 2016.

[16] Z. Zhang, J. Cao, and D. Zhou, "Novel LMI-based condition on global asymptotic stability for a class of Cohen-Grossberg BAM networks with extended activation functions," IEEE Transactions on Neural Networks and Learning Systems, vol. 25, no. 6, pp. 1161-1172, 2014.

[17] L. Shi, H. Zhu, S. Zhong, and L. Hou, "Globally exponential stability for neural networks with time-varying delays," Applied Mathematics \& Computation, vol. 219, no. 21, pp. 10487-10498, 2013.

[18] C. Huang, P. Chen, Y. He, L. Huang, and W. Tan, "Almost sure exponential stability of delayed Hopfield neural networks," Applied Mathematics Letters, vol. 21, no. 7, pp. 701-705, 2008.

[19] L. Liu and Q. Zhu, "Almost sure exponential stability of numerical solutions to stochastic delay Hopfield neural networks," Applied Mathematics \& Computation, vol. 266, pp. 698-712, 2015.

[20] J. Pan, X. Liu, and W. Xie, "Exponential stability of a class of complex-valued neural networks with time-varying delays," Neurocomputing, vol. 164, pp. 293-299, 2015.

[21] D. Yang, G. Qiu, and C. Li, "Global exponential stability of memristive neural networks with impulse time window and time-varying delays," Neurocomputing, vol. 171, pp. 1021-1026, 2016.

[22] Q. Zhu and X. Li, "Exponential and almost sure exponential stability of stochastic fuzzy delayed Cohen-Grossberg neural networks," Fuzzy Sets \& Systems, vol. 203, pp. 74-94, 2012.

[23] X. Y. Lou and Q. Ye, "Input-to-state stability of stochastic memristive neural networks with time-varying delay," Mathematical Problems in Engineering, vol. 2015, Article ID 140857, 8 pages, 2015.

[24] A. F. Filippov, "Classical solutions of differential equations with multi-valued right-hand side," SIAM Journal on Control, vol. 5, pp. 609-621, 1967.

[25] Q. Zhu and J. Cao, "Mean-square exponential input-to-state stability of stochastic delayed neural networks," Neurocomputing, vol. 131, pp. 157-163, 2014.

[26] Y. Song, W. Sun, and F. Jiang, "Mean-square exponential inputto-state stability for neutral stochastic neural networks with mixed delays," Neurocomputing, vol. 205, pp. 195-203, 2016. 


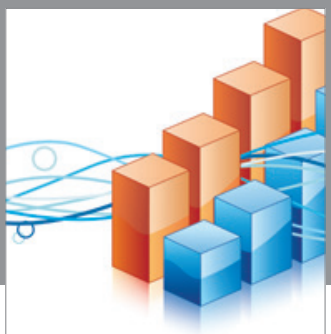

Advances in

Operations Research

vatem alat4

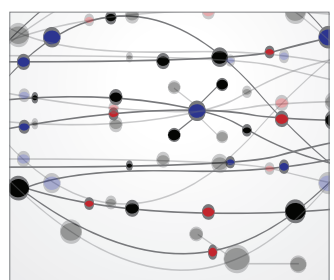

\section{The Scientific} World Journal
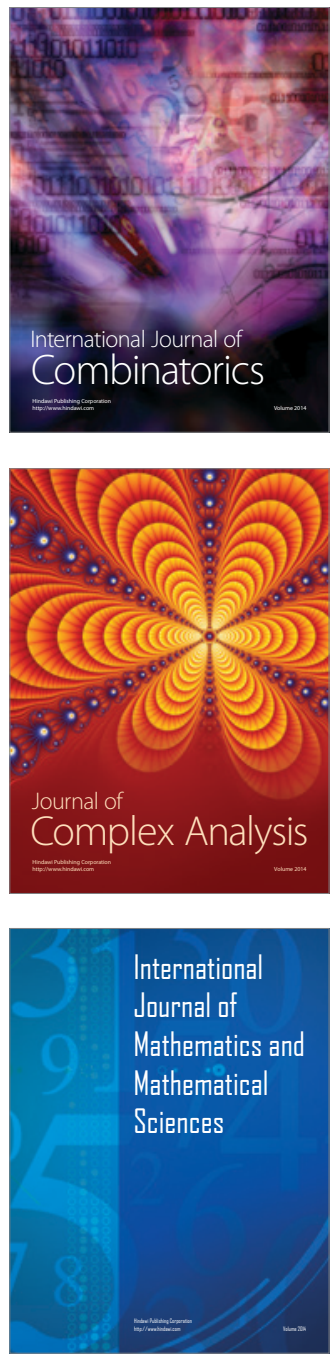
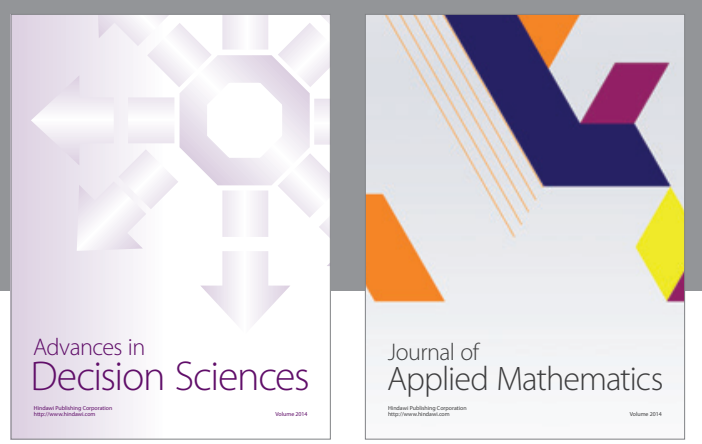

Algebra

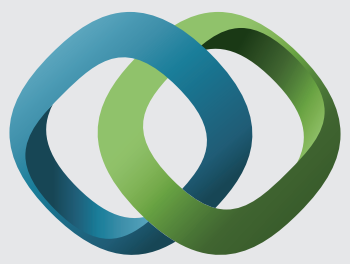

\section{Hindawi}

Submit your manuscripts at

https://www.hindawi.com
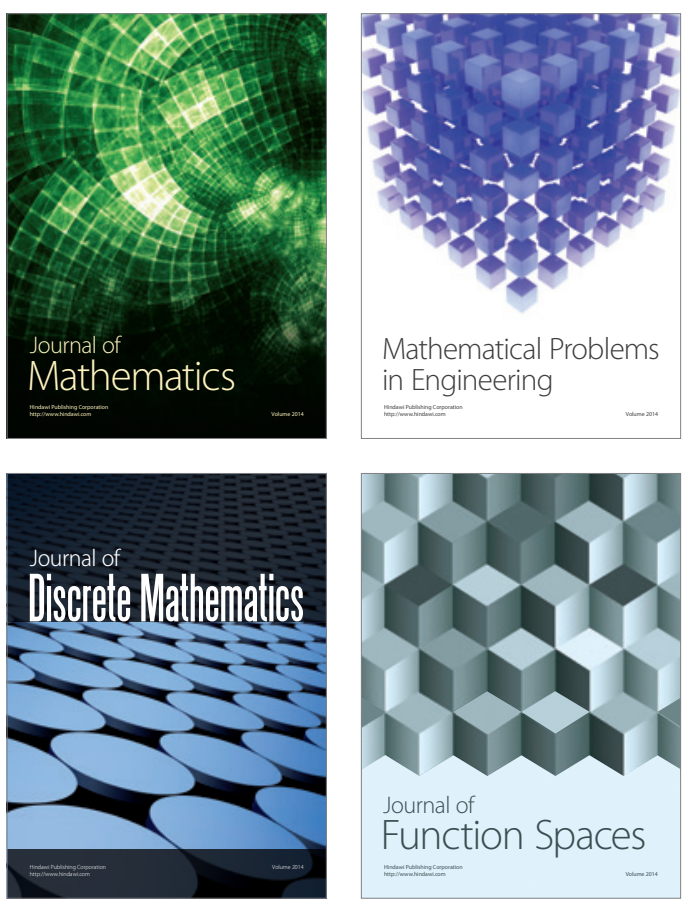

Mathematical Problems in Engineering
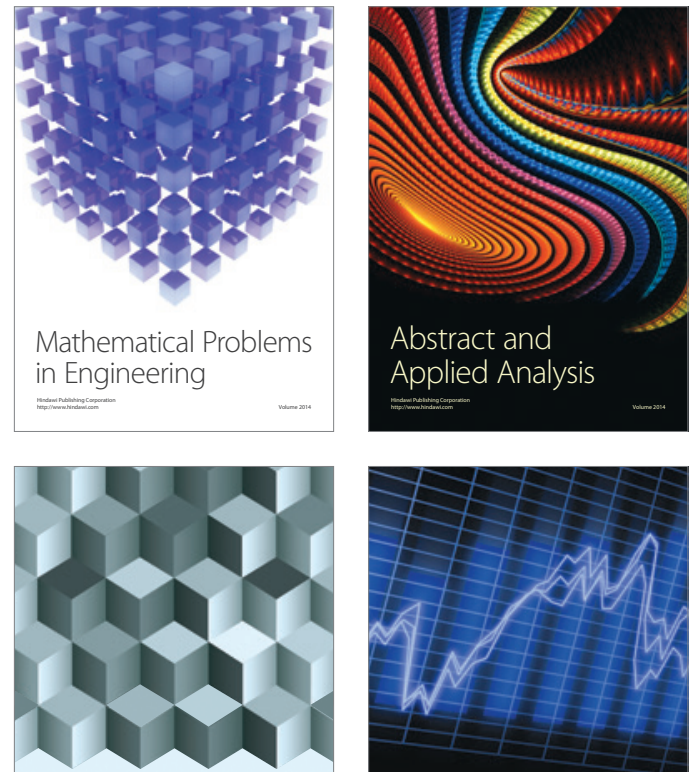

Journal of

Function Spaces

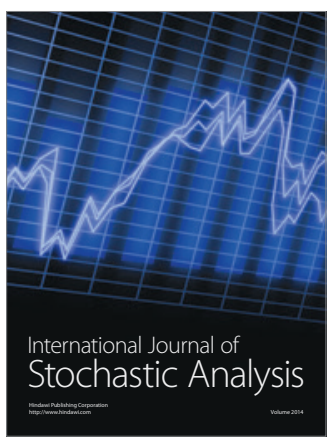

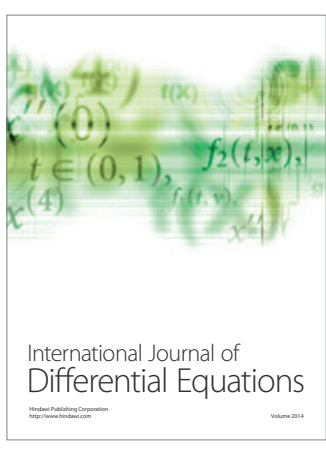
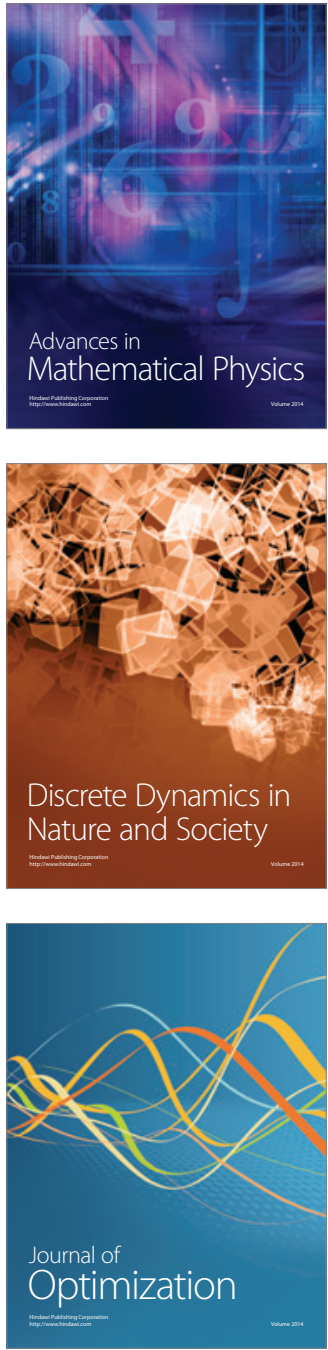